2010-01-01

\title{
Electrochemical Characterisation of Poly Arylene Vinylenes
}

\author{
Jonathan Moghal \\ Technological University Dublin \\ Patrick Lynch \\ Technological University \\ Mary McNamara \\ Technological University, Mary.McNamara@tudublin.ie
}

See next page for additional authors

Follow this and additional works at: https://arrow.tudublin.ie/materart

Part of the Physical Sciences and Mathematics Commons

\section{Recommended Citation}

Moghal, J., Lynch, P. \& McNamara, M. (2010). Electrochemical Characterisation of Poly Arylene Vinylenes. Journal of Electroanalytical Chemistry. vol. 650, no. 1, pg. 159-162. doi:10.1016/j.jelechem.2010.08.002

This Article is brought to you for free and open access by the Materials Synthesis and Applications at ARROW@TU Dublin. It has been accepted for inclusion in Articles by an authorized administrator of ARROW@TU Dublin. For more information, please contact arrow.admin@tudublin.ie, aisling.coyne@tudublin.ie, gerard.connolly@tudublin.ie. 
Authors

Jonathan Moghal, Patrick Lynch, Mary McNamara, Hugh Byrne, and Gordon Chambers

This article is available at ARROW@TU Dublin: https://arrow.tudublin.ie/materart/3 
Journal of Electroanalytical Chemistry

Electrochemical characterisation of poly arylene vinylenes

J. Moghal ${ }^{a}$, P. Lynch ${ }^{b}$, M. McNamara ${ }^{b}$, H.J. Byrne ${ }^{b}$, G. Chambers ${ }^{a, b}$

a School of Physics/FOCAS Research Institute, Dublin Institute of Technology,

Kevin St., Dublin 8, Ireland

b School of Chemistry/FOCAS Research Institute, Dublin Institute of Technology,

Kevin St., Dublin 8, Ireland

\section{Abstract}

The electrochemical properties of a newly synthesised set of PPV derivatives were measured by cyclic voltammetry. A new octyloxy substituted PPV derivative was first synthesised (POPV). The structure of the POPV is varied systematically by the replacement of the phenyl units by naphthyl and anthryl units and the effect of these variations on the electrochemical properties is explored. The HOMO-LOMO levels of the polymers were determined from the onset potentials for the $\mathrm{p}$-doping and $\mathrm{n}$-doping scans. From this the electrochemical bandgap for each of the polymers was calculated. The systematic change in the structure of the POPV results in a systematic increase in the LOMO level of the polymer series whereas a concomitant increase in HOMO level results in an overall increase in the bandgap of the polymers series. The variation in the electrochemical, optical properties and vibrational properties observed within the polymer series were all found to correlate with the structural changes made, pointing towards structure property relationships and a guide to future synthetic strategies.

\section{Introduction}

Conducting polymers, such as polyacetylenes, polyaniline and polythiophene have a high degree of p-orbital conjugation. The discovery of electroluminescence and charge storage in poly (p-phenylenevinylene) (PPV) [1] has led to extensive research in PPV and its derivatives due to their potential application as emissive layers in light-emitting diodes (LEDs) [2,3] and donor materials in organic photovoltaic devices [4]. The possibility to control the sequence of the alternating units and side chains of the conjugated polymers also allows manipulation of the optical and electronic properties of the materials. In order to optimize polymer properties for device application it is essential to obtain a detailed knowledge of their redox behavior [5].

Conjugated polymers are capable of being electrochemically oxidized or reduced by the withdrawal or injection of electrons. When in an oxidized state, the negatively charged ions move (doped) into the polymer to keep electroneutrality (p-doping); while when the polymer is in the reduced state, positively charged cations are doped into the polymer film (n-doping) [6]. The doped and undoped 
polymers have completely different properties. Most importantly, the doped polymer has a high conductivity, which can be comparable to that of metal [6], while the undoped polymer is a semiconductor or insulator. Cyclic voltammetry (CV) is an important electrochemical technique for measuring the reduction and oxidation potentials of conjugated polymers. Electron affinities, ionisation potentials and electrochemical bandgaps can all be calculated from a cyclic voltammogram [5]. The oxidation peak corresponds to the removal of electrons from the highest occupied molecular orbital (HOMO) and the energy can be related to the ionisation potential whereas the reduction peak corresponds to the electron addition to the lowest occupied molecular orbital (LOMO) and can be related to the electron affinity. The difference between the two can be used to work out the electrochemical bandgap of the polymers [7].

The results of electrochemical investigations of thin films, of a relatively new set of PPV derivatives will be presented. Fig. 1 shows the structures of these PPV derivatives [8]. An octyloxy substituted PPV derivative was first synthesised POPV (1) [8]. The structure of the polymer is varied and the effect of these variations on the electrochemical properties is explored. The introduction of naphthyl units begins firstly by replacing alternate phenyl units with naphthyl units to yield POPV-ONV (2) and finally all phenyl units were replaced to produce a fully conjugated naphthyl polymer, PONV (3) [8]. Anthryl units were then introduced into the polymer. This was done in the same manner as the naphthalene structures, first by replacing alternate phenyl units of the POPV to produce POPVOAV (4) and then by replacing alternate naphthyl units with anthryl units to produce PONV-OAV (5) [8].

The polymers have been extensively characterised in their molecular form [9-11] and it has been seen that their bandgaps and photophysical properties can be related to the systematic structural changes. Of interest here is whether the electrochemical properties are similarly well defined and thus can be systematically tailored for device applications.

\section{Experimental}

Synthesis of the novel polymers have been published elsewhere [8]. Cyclic voltammetry measurements of the PPV derivatives were carried out using a three-electrode cell consisting of a glassy carbon working electrode $(3 \mathrm{~mm}$ diameter), a platinum wire counter electrode and a non-aqueous $\mathrm{Ag} / \mathrm{AgNO} 3$ $(0.10 \mathrm{M})$ reference electrode. The polymer films were prepared by first dissolving the PPV derivatives in chlorobenzene and then drop-casting a film onto the glassy carbon electrode. The electrode was allowed to dry in a controlled atmosphere. The starting potential for the voltammograms was $0 \mathrm{~V}$ and the end point was adjusted in accordance with polymer reduction and oxidation potentials. The p-doing and ndoping were performed separately. Memory effect, relaxation effect, or anomalous electrochemical and overload effects can be neglected as a pre-treatment. There are five current ranges on the Autolab 
software. During the $5 \mathrm{~s}$ pre-treatment the program selects the appropriate current range so as not to cause the working electrode potential to overload. The supporting electrolyte was $0.1 \mathrm{M}$ tetrabutylammonium hexafluorophosphate (TBAPF6 $\mathrm{CH} 3 \mathrm{CN}$ ) in acetonitrile (purchased from Sigma Aldrich) solution. All measurements were performed using a PGSTAT12 Autolab potentiostat with a scan rate of $10 \mathrm{mV} / \mathrm{s}$. All solutions were purged with nitrogen before measurements.

\section{Results and discussion}

\subsection{Cyclic voltammetry of polymer series}

The cyclic voltammograms of the PPV derivatives are shown in Fig. 2. Charge transport is controlled by the diffusion of counterions in the polymer films. For the p-doping scans the POPV, PONV and the PONA-OAV all exhibit a quasireversible electrochemical response [12]. The POPV-ONV and the POPV-OAV show strong oxidation peaks but as there is very little quasi reduction in the POPV-ONV and POPV-OAV, hence these have an irreversible electrochemical responses under p-doping conditions. In each case if the potential were increased past the oxidation peak of each polymer, the electroactivity of the polymers was lost permanently due to over oxidation [13]. The polymers can be compared to poly (2-methoxy-5-(20-ethylhexyloxy)-1,4 phenylenevinylene) (MEHPPV). MEH-PPV has been intensely studied over the last decade for application in organic LED [14-16] and photovoltaic devices [17-19]. The electrochemical properties of MEH-PPV have been similarly well documented $[13,20,21]$. CV performed on MEHPPV showed that the oxidation potential for the p-doping scan occurred at $0.62 \mathrm{~V}$. The oxidation potentials of the polymers presented here are greater than that of MEH-PPV. The increase in the oxidation potential implies that in a solid-state device the polymers should possess good hole blocking properties [22]. Table 1 summarises the oxidation and reduction potentials of MEH-PPV and the polymer series. The n-doping or reduction scans of POPV, POPV-ONV and PONV-OAV were all shown to be quasi reversible, where as the PONV and POPV-OAV scans were fully reversible [13]. POPV and the POPV-ONV polymers did not produce uniform thin films on the electrode. The shoulders at $0.55 \mathrm{~V}$ and $1.6 \mathrm{~V}$ in those polymer scans are attributed to the underlying glassy carbon electrode and not a reduction process. The replacement of the phenyl unit with the naphthyl unit and the addition of the naphthyl or anthryl to the backbone resulted in a decrease in the reduction potential. The current peaks in the POPV-ONV n-doping scan are much narrower than the rest of the polymer series [23]. This indicates that the amount of charge consumed is less compared to the rest of the series. The onset potentials of the p-doping and n-doping scans can be used to determine the LOMO (electron affinity, EA) and HOMO (ionisation potential, IP) energy levels of the polymers [23] and the potential difference can be used to estimate the band gap of the polymer. De Leeuw et al. predicted that by adding $4.4 \mathrm{~V}$ [24] to the onset potentials, the energy levels in electron volts corresponding to the 
electrochemical potentials (vs. SCE) could be obtained. This relationship can be mathematically described using the following relations [7,24]:

$1 / 2$ Eonset_ox $1 / 4$ Ionisation Potential ठIPP_ 4:4 ठ1P

$1 / 2$ Eonset_red $1 / 4$ Electron Affinity ठEAP_ $4: 4$ ठ2P

where $1 / 2 \bar{E}$ Enset_ox and $1 / 2$ Eonset red are the onset potentials for the oxidation and reduction of the polymers $[7,23,24]$. The onset potentials $1 / 2$ Eonset_ox and $1 / 2$ Eonset_red were calculated from the intersection of the two tangents drawn at the rising oxidation (reduction) current and background current in the cyclic voltammogram [23]. Table 1 summarises the calculated results from the polymers. The optical bandgaps of the conjugated polymers as measured by UV/VIS absorption spectroscopy were found to be in close approximation of the electrochemical bandgaps.

\subsection{Structure property relationship of HOMO-LOMO level}

The electronic properties of the homologous polymer series have been shown to be a balance of the increased electron density of the aromatic substituents and their communication across the vinyl bond [9]. The phenyl units have been shown to have a strong electron affinity of $\_1.12 \mathrm{eV}$, compared to that of the naphthyl (electron affinity $=\_0.19 \mathrm{eV}$ ) units and the anthryl units (electron affinity $=+0.53 \mathrm{eV}$ ) [25]. The addition of the naphthyl units to the phenyl units (POPVONV) results in a depletion of the $p$ electron density of the vinyl bond which has been shown to be manifest for example in a systematic softening of the vinyl stretch band in the Raman spectrum [9]. This governs the systematic variation of the optical bandgap and should therefore govern the systematic variation in the LOMO. The electron density of the vinyl bridging unit has been quantified in terms of the parameter EAvinyl [9]. Fig. 3 shows a plot of the LOMO levels of the polymers against the EAvinyl parameter. EAvinyl is an accurate variation of the electronic character of the vinyl bond as the larger, higher electron affinity units, are substituted into the chain. A polymer, being a chain-like structure, will be limited by the weakest link and thus the limiting of the conjugation across the vinylene bond will hinder the electron-phonon coupling being distributed across the entire polymer backbone. From Fig. 3 a linear relationship between the EAvinyl parameter and the LOMO levels can be deduced. Further comparison can be made between the calculated HOMO levels of the polymers and the optical bandgap. Fig. 4 shows a plot of the calculated HOMO levels for the polymers against their optical bandgap. Although an exact linear dependence is not inferred, a correlation is clearly evident and a line is plotted as a guide to observe the trend. Although an increase in HOMO level is favourable for hole blocking properties in a device structure, it can also be seen from Fig. 4 that the increase in HOMO level results in an increase in the bandgap of the polymers.

\section{Conclusion}

Electrochemical oxidation and reduction potentials of newly synthesised PPV derivatives were measured using cyclic voltammetry. The structural changes to 
the original polymer POPV, affected the doping potentials. The introduction of anthracene and naphthylene to the POPV results in the doping potentials being shifted to a lower potential. When compared to MEH-PPV, it can be seen that for the p-doping scans the oxidation potential of the polymers was increased. This indicated that the polymers should possess good hole blocking properties, if used in a solid-state device [9]. In the n-doping scans the POPV, POPV-ONV, POPV OAV and the PONV-OAV had a larger reduction potential than MEHPPV, whereas the PONV had a lower reduction potential than that of MEH-PPV. Therefore the POPV, POPV-ONV, POPV-OAV and the PONV-OAV would be more difficult to reduce than MEH-PPV, but the PONV would be reduced more easily than MEH-PPV. The polymers all showed good reversibility for the ndoping scans. The polymers may be potential candidates for photovoltaic cells and LED devices. The variations in the electrochemical, optical and vibrational properties observed within the polymer series were all found to correlate with structural changes made, pointing towards structure property relationships and a guide to future synthetic strategies.

\section{References}

[1] J.H. Burroughes, D.D.C. Bradley, A.R. Brown, R.N. Marks, K. Mackay, R.H. Friend, P.L. Burn, A.B. Holmes, Nature 347 (1990) 539-541.

[2] R.H. Friend, R.W. Gymer, A.B. Holmes, J.H. Burroughes, R.N. Marks, C. Tiliani, D. Bradley, D.A. Santos, J.L. Bredas, M. Logdlund, W. Salaneck, Nature (London)397 (1999) 121-127.

[3] H.K. Shim, J.I. Jin, Adv. Polym. Sci. 158 (2002) 194-244.

[4] N.S. Sariciftci, L. Smilowitz, A.J. Heeger, F. Wudl, Science 258 (1992) 14741476.

[5] M. Helbig, H.-H. Horhold, Makromol. Chem. 194 (1993) 1607-1618.

[6] I. Kosuke, Electrochemistry in Nonaqueous Solutions, Wiley-VCH Verlag GmbH \& Co. KgaA, 2002.

[7] R. Cervini, X.C. Li, G.W.C. Spencer, A.B. Holmes, S.C. Moratti, R.H. Friend, Synth. Met. 84 (1997) 359-369.

[8] P. Lynch, L. O’Neill, M. McNamara, H.J. Byrne, Macromolecules 40 (2007) 7895-7901.

[9] L. O’Neill, P. Lynch, M. McNamara, H.J. Byrne, J. Phys. Chem. A 111 (2007) 7999-8005.

[10] L. O'Neill, P. Lynch, M. McNamara, H.J. Byrne, Phys. Chem. A 111 (2) (2007) 299-305. 
[11] L. O'Neill, P. Lynch, M. McNamara, H.J. Byrne, Polymer 49 (2008) 41094114.

[12] C.M.A. Brett, A.M.O. Brett, Electrochemistry Principles, Methods and Applications, Oxford Science Publications, 1993.

[13] M.M. Richter, R.F. Fan, F. Klavetter, A.J. Heeger, A.J. Bard, Chem. Phys. Lett. 226 (1-2) (1994) 115-120.

[14] D. Braun, A.J. Heeger, Appl. Phys. Lett. 58 (18) (1991) 1982-1984.

[15] I.D. Parker, J. Appl. Phys. 75 (3) (1994) 1656-1666.

[16] J.C. Scott, J.H. Kaufman, P.J. Brock, R. DiPietro, J. Salem, J.A. Goitia, J. Appl. Phys. 79 (5) (1996) 2745-2751.

[17] N.S. Sariciftci, D. Braun, C. Zhang, V.I. Srdanov, A.J. Heeger, G. Stucky, F. Wudl, Appl. Phys. Lett. 62 (6) (1993) 585-587.

[18] M.G. Harrison, J. Gruener, G.C.W. Spencer, Phys. Rev. B 55 (12) (1997) 7831-7849.

[19] J.J. Dittmer, K. Petritsch, A.E. Marseglia, R.H. Friend, H. Rost, A.B. Holmes, Synth. Met. 102 (1999) 879-880.

[20] C. Yang, G. He, R. Wang, Y. Li, J. Electroanal. Chem. 471 (1999) 32-36.

[21] A.L. Holt, J.M. Leger, S.A. Carter, J. Chem. Phys. 123 (2005) 044704-7.

[22] X.C. Li, G.C.W. Spencer, A.B. Holmes, S.C. Moratti, F. Cacialli, R.H. Friend, Synth. Met. 76 (1996) 153-156.

[23] Y. Li, Y. Cao, J. Gao, D. Wang, G. Yu, A.J. Heeger, Synth. Met. (99) (1999) $243-248$.

[24] D.M. de Leeuw, M.M. J Simenon, A.R. Brown, R.E.F. Einerhand, Synth. Met. 87 (1997) 53-59.

[25] J.C. Rienstra-Kiracofe, C.J. Barden, S.T. Brown, H.F. Schaefer, J. Phys. Chem. A 105 (2001) 524-528. 